University of Texas Rio Grande Valley

ScholarWorks @ UTRGV

$10-2021$

\title{
Endovascular thrombectomy in patients with large core ischemic stroke: a cost-effectiveness analysis from the SELECT study
}

\author{
Amrou Sarraj \\ Elena Pizzo \\ Kyriakos Lobotesis \\ James C. Grotta \\ Ameer E. Hassan \\ The University of Texas Rio Grande Valley
}

See next page for additional authors

Follow this and additional works at: https://scholarworks.utrgv.edu/som_pub

Part of the Neurosciences Commons, and the Surgery Commons

\section{Recommended Citation}

Sarraj, A., Pizzo, E., Lobotesis, K., Grotta, J. C., Hassan, A. E., Abraham, M. G., Blackburn, S., Day, A. L., Dannenbaum, M. J., Hicks, W., Vora, N. A., Budzik, R. F., Sharrief, A. Z., Martin-Schild, S., Sitton, C. W., Pujara, D. K., Lansberg, M. G., Gupta, R., Albers, G. W., Kunz, W. G., ... SELECT Investigators (2021). Endovascular thrombectomy in patients with large core ischemic stroke: a cost-effectiveness analysis from the SELECT study. Journal of neurointerventional surgery, 13(10), 875-882. https://doi.org/10.1136/ neurintsurg-2020-016766

This Article is brought to you for free and open access by the School of Medicine at ScholarWorks @ UTRGV. It has been accepted for inclusion in School of Medicine Publications and Presentations by an authorized administrator of ScholarWorks @ UTRGV. For more information, please contact justin.white@utrgv.edu, william.flores01@utrgv.edu. 


\section{Authors}

Amrou Sarraj, Elena Pizzo, Kyriakos Lobotesis, James C. Grotta, Ameer E. Hassan, Michael G. Abraham, Spiros Blackburn, Arthur L. Day, Mark J. Dannenbaum, and William Hicks 
Endovascular Thrombectomy in Patients with Large Core Ischemic Stroke: A CostEffectiveness Analysis from the SELECT Study

Authors:

Amrou Sarraj MD', Elena Pizzo PhD², Kyriakos Lobotesis MD³ , James Grotta MD, Ameer Hassan DO ${ }^{5}$, Michael Abraham MD ${ }^{6}$, William Hicks MD ${ }^{7}$, Nirav Vora MD ${ }^{7}$, Ronald

Budzik MD ${ }^{7}$, Spiros Blackburn MD ${ }^{8}$, Arthur Day MD ${ }^{8}$, Mark Dannenbaum MD ${ }^{8}$, Peng R Chen $\mathrm{MD}^{8}$, Anjail Sharrief MD' ${ }^{1}$, Clark Sitton MD ${ }^{9}$, Rishi Gupta MD $^{10}$, Sean Savitz MD ${ }^{11}$, Gregory W Albers $\mathrm{MD}^{12}$, Wolfgang Kunz MD ${ }^{13}$

${ }^{1}$ The University of Texas at Houston, Neurology, Houston, Texas, USA.

${ }^{2}$ Department of Applied Health Research, University College London, London, UK.

${ }^{3}$ Imaging Department, Imperial College Healthcare NHS Trust, Charing Cross Hospital, London, UK.

${ }^{4}$ Memorial Hermann Hospital - Texas Medical Center, Clinical Institute for Research and Innovation, Houston, USA

${ }^{5}$ Department of Neurology, University of Texas Rio Grande Valley, Harlingen, USA

${ }^{6}$ University of Kansas Medical Center, Neurology, Kansas City, Kansas, USA

${ }^{7}$ OhioHealth-Riverside Methodist Hospital, Neurology, Columbus, USA.

${ }^{8}$ The University of Texas at Houston, Neurosurgery, Houston, USA.

${ }^{9}$ The University of Texas at Houston, Neuroradiology, Houston, USA.

${ }^{10}$ Wellstar Health System, Neurology, Atlanta, USA

${ }^{11}$ The University of Texas Institute of Stroke and Cerebrovascular Diseases, Neurology, Houston, Texas, USA.

${ }^{12}$ Stanford University, Neurology, Stanford, USA.

${ }^{13}$ University Hospital, LMU Munich, Radiology, Munich, Germany

\section{Corresponding Author:}

Dr. Amrou Sarraj

UT McGovern Medical School,

6431 Fannin St, MSB 7.044; Houston, TX - 77030

Phone number: 713-500-7857 Fax: 713-500-0638 
Email address: Amrou.Sarraj@uth.tmc.edu

Cover Title: Cost-Effectiveness of Thrombectomy in Large Core

Tables: 3

Figures: 3

Total Word Counts: 6002 


\title{
Endovascular Thrombectomy in Patients with Large Core Ischemic Stroke: A Cost- Effectiveness Analysis from the SELECT Study
}

\begin{abstract}
:
Background: Whether endovascular thrombectomy (EVT) is cost-effective in large ischemic core infarcts is unknown.

Methods: In the prospective multicenter cohort study of imaging selection study (SELECT), large core was defined as CT ASPECTS $<6$ or CTP ischemic core volume $(\mathrm{rCBF}<30 \%) \geq 50 \mathrm{cc}$. A Markov model estimated costs, quality-adjusted life years (QALYs) and the incremental costeffectiveness ratio (ICER) of EVT compared to medical management (MM) over life time. The lower and upper willingness to pay (WTP) per QALY were set at $\$ 50,000$ and $\$ 100,000$ and the net monetary benefit (NMB) for EVT were calculated. Probabilistic sensitivity analysis (PSA) and cost-effectiveness acceptability curves (CEAC) were assessed for EVT in SELECT and other pivotal EVT trials.
\end{abstract}

Results: From a prospective cohort trial of 361 patients, 105 had large core on CT or CTP (EVT 62, MM 43). 19 (31\%) EVT patients achieved mRS 0-2 vs 6 (14\%) MM patients (aOR: 3.27, 95\% CI: $1.11-9.62 ; P=.03$ ) with a shift towards better mRS (adj cOR: $2.12,95 \%$ CI: $1.05-4.31$, $P=.04)$. Over the projected lifetime of stroke patients presenting with large ischemic core, EVT was associated with incremental costs of $\$ 33,094$ and a gain of 1.34 QALYs per patient, resulting in ICER of $\$ 24,665$ per QALY. EVT has a higher NMB compared to MM at the lower (EVT: $-\$ 42,747$ vs MM: $-\$ 76,740$ ) and upper (EVT: $\$ 155,041$ vs MM: $\$ 57,134)$ thresholds of willingness to pay. The PSA confirmed the results and the CEAC showed $77 \%$ and $92 \%$ cost- 
effectiveness probability of EVT at the WTP of $\$ 100,000$, respectively. EVT was associated with an increment of $\$ 28,962$ in societal costs. The Pivotal EVT trials (HERMES, DAWN and DEFUSE 3) were dominant in a sensitivity analysis at the same inputs, with societal cost savings of $\$ 38,072, \$ 86,358$ and $\$ 22,837$, respectively.

Conclusion: EVT may result in better outcomes in large core patients with higher QALYs, NMB and high cost-effectiveness acceptability rates based on current WTP thresholds.

Clinical Trial Registration: http://www.clinicaltrials.gov. Unique identifier: NCT02446587 


\section{Introduction}

Stroke is a global health problem, with more than 13 million new strokes and 5.5 million deaths every year ${ }^{1}$. In the USA, every year 795,000 individuals suffer from new or recurrent stroke, resulting in 140,000 deaths $^{2}$. Stroke is also the leading cause of serious long-term disability, resulting in 116.5 million quality-adjusted life year (QALY) $\operatorname{lost}^{1}$ worldwide every year. Moreover, it is an expensive disease to treat and manage with annual direct costs of $\$ 28$ billion and indirect costs of $\$ 17.5$ billion on the US economy, with a projected increase in direct costs to $\$ 94.3$ billion by year $2035^{2}$. Effective stroke treatments that improve clinical outcomes and reduce disability may help reduce the economic burden of stroke in society and in our health care systems by reducing direct and indirect costs.

Endovascular thrombectomy (EVT) has been established as a transformative treatment for ischemic stroke patients with large vessel occlusion; safety and efficacy have been well documented in patients with small ischemic core infarcts, both in early ${ }^{3-7}$ and late ${ }^{8,9}$ time windows. While randomized trials for efficacy and safety of EVT in patients with large ischemic stroke are ongoing ${ }^{10-13}$, potential evidence of EVT efficacy and safety in these patients was recently presented from a large, multicenter, prospective cohort data ${ }^{14}$. With prevalence of large core strokes estimated to be as high as $\sim 30 \%{ }^{14}$, these patients are frequently encountered in daily practice. Treating physicians have several considerations while determining the treatment approaches, such as the poor natural history in patients treated with medical management only as compared to the lower likelihood of functional independence and the potential safety concerns with EVT. One of the considerations for the healthcare systems and for society is whether treatment with endovascular thrombectomy is cost-effective. While cost-effectiveness of EVT in 
patients with small ischemic core has been assessed and deemed favorable ${ }^{15-21}$, it remains unknown whether EVT is cost-effective in patients presenting with large ischemic core infarcts. The implications of EVT on societal costs related to large stroke patients have also not been studied in detail.

We sought to assess EVT cost-effectiveness by performing a cost- effectiveness analysis for EVT as compared to medical management only (MM) using outcomes in patients with large ischemic core infarcts on CT (ASPECTS $<6)$, CTP $\left(\mathrm{rCBF}<30 \%\right.$ volume $\left.\geq 50 \mathrm{~cm}^{3}\right)$ or both from the SELECT study, a prospective, multicenter cohort study of imaging selection ${ }^{22}$. This study sub-analysis aims were:

1. To assess the overall gain in QALYs

2. To identify the incremental cost-effectiveness ratio (ICER) for EVT

3. To assess the net monetary benefit (NMB) at various thresholds of willingness to pay (WTP)

4. To compare EVT cost-effectiveness in patients with large ischemic core to those with small core infarcts.

5. To assess EVT societal cost benefits in patients with large ischemic core

\section{Methods:}

\section{Study population:}

"The Optimizing Patient Selection for Endovascular Treatment in Acute Ischemic Stroke (SELECT): a Prospective Multicenter Cohort Study of Imaging Selection" ${ }^{22}$ enrolled patients with stroke attributable to large-vessel occlusion who were treated with endovascular 
thrombectomy plus medical management or medical management alone, based on CT or CTP findings, up to 24 hours from the point that they were last known to be well at 9 comprehensive stroke centers across the United States. The decision to proceed with EVT vs medical management alone was made at the discretion of the local investigators in a nonrandomized fashion. The study initially included patients up to 8 hours after stroke onset, with the enrollment window extended up to 24 hours after the results of the Clinical Mismatch in the Triage of Wake Up and Late Presenting Strokes Undergoing Neurointervention With Trevo (DAWN) trial $^{8}$ were announced. Patients with large ischemic cores were defined as having an ASPECTS of 5 or less on non-contrast CT or an ischemic core volume of $50 \mathrm{~cm}^{3}$ or more on CTP based on the volume of tissue with a relative cerebral blood flow less than $30 \%$ at presentation.

\section{Cost-Effectiveness Analysis:}

We performed a cost-effectiveness analysis to compare costs and outcomes of endovascular thrombectomy (EVT) in addition to medical management (MM) compared to MM only in patients with large core acute ischemic stroke. The outcome measures were quality-adjusted life years (QALYs). QALYs are assessed using the survival time and the utility associated to the modified Rankin Scale (mRS) score. For each $\mathrm{mRS}$ score we used utility values obtained from a recent consensus analysis. The analysis took the health care service provider perspective, including only the costs of direct medical treatment (hospital admissions, ICU, rehabilitation). An analysis from societal perspective was also attempted that accounts for the societal costs (as in productivity losses, informal care and private cost for patients). Costs were calculated in 2017 US\$, inflated where necessary ${ }^{23}$. The time horizon was selected to be over lifetime. All costs and outcomes after the first year were discounted at an annual rate of $3 \%$. 


\section{Model Structure:}

We created a decision analysis model to assess cost-effectiveness of EVT using TreeAge Healthcare Pro version 2020 (TreeAge, Williamstown/MA). The treatment arms include best MM (including the use of IV tPA where applicable) without subsequent EVT vs best MM followed by EVT. The decision model consisted of two different models 1) a short run model to assess the transitions measured at 90 days following stroke and 2) a long run model to assess transitions over the course of lifetime following the initial stroke, beginning at 90-day post stroke. The short run model analyzed the clinical outcomes and costs associated with the treatment at 90-day interval following the index stroke. The long run model was created to estimate the transitions in clinical outcomes and associated post-stroke costs with cycles of 1 year. At the end of each cycle, the patient could remain in the same health state, experience a recurrent stroke and recover to the same state or transition to other states or die from stroke or other causes. Outcomes were based on modified Rankin Scale (mRS) scores measured at 90 days after stroke. $^{24}$

\section{Model Input Parameters}

The initial probabilities for short run model to analyze 90 day clinical outcomes were identified from the large core cohort of the SELECT study ${ }^{14}$. The study reported outcomes of 105 patients (62 EVT, $43 \mathrm{MM}$ ) with large ischemic stroke on non-contrast CT, CT perfusion or both; demonstrating functional independence rates of $31 \%$ and $14 \%$ in patients treated with EVT and MM respectively. For comparative models for patients with small core, results provided in HERMES metanalysis ${ }^{25}$ were used for short run models in early time window (0-6 hours), DAWN ${ }^{8}$ and DEFUSE $3^{9}$ results were used for trials beyond 6 hours of stroke onset. The 
transitional probabilities beyond 90 days of stroke onset were populated using historical data from multiple prospective cohort studies, identifying probabilities of recurrent stroke, death and changes in functional status over the course of time for patients suffering from index stroke. ${ }^{26-28}$ The measures for utilities at different mRS categories were obtained from a prospectively validated cohort evaluating EuroQol in post-stroke patients. ${ }^{29}$ Table 1 provides further information regarding the parameters used in the decision analysis model.

\section{Treatment Costs}

Data was used from the National Inpatient Sample $^{30}$ and data available in the literature ${ }^{31}$ to estimate costs and we inflated as necessary using the inflation rate from national sources ${ }^{24}$. The costs are presented in Table 1.

The additional cost of IV thrombolysis was estimated to be $\$ 6,961$, including the cost of the therapy, the medication and administration from the National Inpatient Sample ${ }^{30}$, inflated at the 2017 US \$.

The cost of the EVT varied in each trial was estimated to be $\$ 14,454$. This includes the cost of the devices (stent-retreivers), materials and intervention.

The health care costs in the 3 months following a stroke include the acute management (length of stay in the acute stroke unit, in the acute high dependency unit, and in the rehabilitation ward, as well as the supported discharge cost and community care costs) and rehabilitation costs. ${ }^{32}$ The ongoing costs in the following years include rehabilitation costs and follow-up costs. ${ }^{31}$ Both acute and ongoing costs were applied based on the disability observed at 90-day on modified Rankin Scale score in 7 different categories. The cost of a recurrent stroke was estimated as the mean expected cost to treat an average stroke that does not need MM or EVT. ${ }^{33}$ 


\section{Initial and Transitional Probabilities}

The initial probabilities were defined based on modified Rankin Scale score at 90 days and were assessed using results from the SELECT study for patients presenting with large ischemic core for the main results. The initial probabilities for comparison models with the trials were assessed from the results published from the DAWN ${ }^{8}$ and DEFUSE 3 trials ${ }^{9}$ and HERMES metaanalysis $^{25}$. The transitional probabilities were defined as the probability to move from one health state to another after 90 days results and were implemented in annual cycle for the long run model for a horizon of lifetime. The age specific annual death rates were obtained from United States Life Tables. ${ }^{27}$ Excess deaths because of stroke were incorporated as additional hazard ratios identified from the contemporary cohort studies. ${ }^{28}$ The other transitional probabilities were also obtained from various large prospective cohorts. ${ }^{26}$

\section{Cost-Utility Measures and Outcomes:}

The cost-effectiveness of EVT was expressed in terms of its incremental cost-effectiveness ratio (ICER), defined as the ratio of the difference in the costs between EVT and MM and gain in Qality Adjusted Life Years between the treatments demonstrated by the model.

$$
I C E R=\frac{\text { Average Cost of } E V T-\text { Average Cost of } M M}{\text { Average QALYS of EVT - Average QALYS of } M M}
$$

Results are also expressed in terms of net monetary benefits (NMB), calculated as the mean QALYs per patient accruing to that treatment multiplied by the willingness to pay (WTP) for a QALY (the cost-effectiveness threshold) minus the mean cost per patient for the treatment. 


\section{Net Monetary Benefit $=[$ Average QALYs with EVT X Willingness to Pay $]-$ Mean Difference in the Cost of EVT}

The lower and upper willingness to pay for NMB calculations were set to $\$ 50,000$ and $\$ 100,000$ per QALY.

\section{Analysis from Societal Perspective:}

To assess the impact of index stroke on overall societal productivity, we used human captial approach to calculate costs associated with lost productivity as well as costs of informal care provided by the family. Lost societal productivity due to premature deaths and post stroke morbidity were calculated separately. Using US Census Bureau ${ }^{34}$ gross wages and age-based employment rates provided by US Bureau of Labor Statistics ${ }^{35}$, lost productivity due to premature mortality was calculated. Furthermore, we assumed complete retirement at age 80. Non-stroke related premature deaths were not considered for these evaluations.

To obtain the lost productivity due to stroke-related morbidity, the aforementioned productivity measures were multiplied by relative earnings of stroke survivors $(82.5 \%)$ and probabilities of return to work (RTW) based on 90-day functional status. The cost of informal care provided by the family members were stratified based on 90-day functional status and calculated using hourly wages for home health aides for year $2017 .{ }^{34}$

\section{Sensitivity Analysis}

A probabilistic sensitivity analysis (PSA) was performed to establish the impact of the uncertainty characterising the input parameters and assess the robustness of the results, using 
10,000 runs for the second order Monte Carlo simulation. A distribution was assigned to each parameter value and a random value from the corresponding distribution was selected to be used as an input for the Markov model. The mean (median) cost, QALYs and NMB for each treatment were calculated from the 10,000 simulations; and the probability that the interventions are costeffective is summarised in the cost-effectiveness acceptability curves (CEACs). We also conducted the same analysis, using the data from randomized trials that proved EVT efficacy and safety in patients with small ischemic core: HERMES ${ }^{25}$, DEFUSE $3^{9}$ and DAWN ${ }^{8}$.

\section{Results}

Functional Outcomes in Patients with Large Ischemic Core Infarcts:

Of 361 patients enrolled in SELECT, 105 (29\%) had a large ischemic core on CT or CTP or both (EVT 62, MM 43). Functional independence (modified Rankin Scale score of 0-2) at 90 days was achieved in $19(31 \%)$ EVT patients vs $6(14 \%) \mathrm{MM}(\mathrm{OR}: 3.27,95 \%$ CI: 1.11-9.62; $P=.03$ ) with a shift towards better mRS (cOR: $2.12,95 \%$ CI: 1.05-4.31, $P=.04)^{14}$. Mortality was observed in 29\% (18/62) of EVT and 42\% (18/43) of MM patients, $\mathrm{p}=0.17$. Neurological worsening (EVT: 13/62 (21\%) vs MM: 8/43 (20\%), p=0.87) and symptomatic ICH (EVT: 8/62 (13\%) vs $3 / 43(7 \%), \mathrm{p}=0.51)$.

\section{Base Case Analysis:}

Using base case values, EVT following MM performed in patients with large ischemic core infarcts was associated with an incremental cost of $\$ 33,094$ per patient over projected lifetime(Table 2). EVT-treated patients could gain 1.34 incremental QALYs. 
The ICER of EVT compared to MM was \$24,665 per QALY. The EVT has a higher NMB compared to MM alone at the lower (EVT: $-\$ 42,747$ vs MM: $-\$ 76,740)$ and upper (EVT: \$155,041 vs MM: \$57,134) thresholds of WTP, indicating that EVT is cost-effective. EVT was also associated with incremental costs of $\$ 28,962$ in an analysis from the societal perspective.

\section{Sensitivity Analysis:}

Figure 2 demonstrates the probabilistic sensitivity analysis based on 10,000 simulations. A significantly higher proportion of results are in the quadrant where an increment in costs is associated with a QALY gain and most of the result are under the \$100,000/QALY threshold line, therefore there is a high probability that EVT is cost-effective. The cost-effectiveness acceptability curves (CEAC) demonstrates the probability that each treatment is cost-effective at different values of the willingness to pay for a QALY (Figure 3). EVT has $77 \%$ \& $92 \%$ probability of being cost-effective compared to MM when the willigness to pay is $\$ 50,000$ and $\$$ 100,000 per QALY, respectively.

\section{Comparison of EVT Cost-Effectiveness in Large Core Infarcts to Patients with Small Core}

\section{Infarcts:}

Overall, data from HERMES meta-analysis in early time window and DAWN and DEFUSE 3 in late time window demonstrated overall higher lifetime costs associated with MM as compared to EVT. Cost-effectiveness analysis based on data from HERMES meta-analysis demonstrated average overall cost savings of $\$ 29,964$ over lifetime in patients treated with EVT, with a gain of 1.62 QALY (i.e. dominant over MM) and almost 100\% probability of EVT being cost-effective in probabilistic sensitivity analysis at lower and upper thresholds of WTP. EVT also decreased 
the societal costs by $\$ 38,072$. Similar results are obtained based on outcomes from DAWN trial (dominant) and DEFUSE 3 trial (dominant) with both the trials demonstrating almost 100\% of being cost-effective at the willingness to pay thresholds of $\$ 50,000$ and $\$ 100,000$. EVT was also associated with a reduction in societal costs by $\$ 86,358$ in DAWN and $\$ 22,837$ in DEFUSE 3 trial. Table 3 demonstrates the comparison of incremental EVT costs, QALYs, ICER and societal cost benefits in SELECT large ischemic core patients as compared to previously published RCTs over the horizon of lifetime.

\section{Discussion:}

We found that EVT may result in more lives saved and improved quality of life in large core patients from the SELECT prospective cohort study. This treatment strategy had an acceptable ICER, a higher NMB and high probabilities of being cost-effective at even the lowest bounds of current willingness-to-pay thresholds for the United States healthcare system.

Our analysis suggests that in patients with large ischemic core on CT or CTP, EVT was associated with 1.34 QALY gained and ICER of \$24,665 for each QALY gained. While it resulted in initial $\$ 33,094$ incremental cost per patient, EVT was associated with more lives saved and improved likelihood of being functionally independent (mRS 0-2), increasing the QALYs.

Our results showed an incremental lifetime cost associated with EVT as compared to prior RCTs that enrolled patients with small core. ${ }^{8,9,25}$ This is to a large part likely due to the high mortality rate in SELECT large core subpopulation that received MM only; $42 \%$ as compared to $19 \%$ in HERMES $^{25}$, in $26 \%$ in DEFUSE $3^{9}$ and $18 \%$ in DAWN ${ }^{8}$. Such a high mortality rate 
paradoxically largely reduces the cost in the MM group. For example, healthcare policy makers recognize that life-saving interventions may increase long-term expenditures, creating counterintuitive results. Prominent examples include the effect of smoking cessation or of several preventive measures, which may increase costs in the long run due to a higher life expectancy of the population. ${ }^{36,37}$

Within the same lines, the social cost was higher in our large group cohort due to the reduced mortality with EVT primarily with similar rates of severe and profound disability (mRS) 4-5 between EVT and MM as compared to a difference within the same mRS grades in prior EVT RCTs. Additionally, the median age for our cohort was higher than prior RCTs which may have resulted in an underestimation of the potential savings attributable to EVT and overall savings including productivity losses and higher societal costs.

Previous studies demonstrated the cost-effectiveness of EVT compared to MM up to 24 hours of stroke in small core infarcts ${ }^{15-21}$ with ICER significantly below the current willingness to pay thresholds in patients included in randomized controlled trials (put references only from RCTs). Since most of these trials excluded patients with large ischemic core, involvement of these patients in such analyses is limited. Prior report ${ }^{18}$ demonstrated cost-effectiveness of EVT in patients with ASPECTS of 0-5 at a higher ICER, however concluded that the estimates may be uncertain because of systemic exclusion of such patients from the trials.

With several ongoing clinical trials assessing the efficacy and safety of EVT in stroke patients with large ischemic core on different imaging modalities (SELECT $2^{13}$, IN EXTREMIS ${ }^{12}$, TESLA $^{11}$, TENSION ${ }^{10}$ ), the randomized evidence is forthcoming in this important sub population, which represents more than $20 \%$ of the patients encountered in daily practice. 
Meanwhile, our cost-effectiveness analysis from the SELECT prospective cohort ${ }^{14}$ suggests that EVT may be cost-effective. These results, while still need to be confirmed based on data from the upcoming RCTs, represent further evidence from economical, societal and quality of life assessment to justify these randomized trials to evaluate intervening in patients with more extensive areas of infarct.

There are limitations to our study that should be considered when interpreting the findings. This includes the lack of randomization and potential selection biases associated with prospective, cohort data. Additionally, we did not have direct economic data from the prospective cohort and thus relied upon the overall payments based on current diagnosis-related group (DRG) for EVT. Another limitation is represented by the variability in the methodology adopted in the different trials of EVT effectiveness in patients with small core infarcts, including the use of different devices and medical treatment to treat stroke. However, we conducted an extensive sensitivity analysis that confirms the results.

The strengths of the presented study include data from a multicentre, prospective cohort study, use of prior validated methods, inclusion of probabilistic sensitivity analysis and comparative analysis with prior RCTs using the same parameters that allows for the direct comparison of cost-effectiveness.

\section{Conclusion:}

EVT may result in better outcomes and more lives saved in large core patients with higher QALYs, NMB and an acceptable ICER. This further justifies a randomized trial for EVT efficacy and safety in patients with large core. 


\section{Declaration of conflicting interests}

The authors declare no conflict of interest.

\section{References}

1. Benjamin EJ, Muntner P, Alonso A, et al. Heart Disease and Stroke Statistics-2019 Update: A Report From the American Heart Association. Circulation. 2019;139(10):e56e528. doi:10.1161/CIR.0000000000000659

2. Stroke Facts | cdc.gov. https://www.cdc.gov/stroke/facts.htm. Accessed May 29, 2020.

3. Berkhemer OA, Fransen PSS, Beumer D, et al. A Randomized Trial of Intraarterial Treatment for Acute Ischemic Stroke. N Engl J Med. 2015;372(1):11-20. doi:10.1056/NEJMoa1411587

4. Saver JL, Goyal M, Bonafe A, et al. Stent-Retriever Thrombectomy after Intravenous tPA vs. t-PA Alone in Stroke. N Engl J Med. 2015;372(24):2285-2295. doi:10.1056/NEJMoa1415061

5. Jovin TG, Chamorro A, Cobo E, et al. Thrombectomy within 8 Hours after Symptom Onset in Ischemic Stroke. N Engl J Med. 2015;372(24):2296-2306. doi:10.1056/NEJMoa1503780

6. Goyal M, Demchuk AM, Menon BK, et al. Randomized Assessment of Rapid Endovascular Treatment of Ischemic Stroke. N Engl J Med. 2015;372(11):1019-1030. doi:10.1056/NEJMoa1414905

7. Campbell BC V, Mitchell PJ, Kleinig TJ, et al. Endovascular Therapy for Ischemic Stroke 
with Perfusion-Imaging Selection. N Engl J Med. 2015;372(11):1009-1018.

doi:10.1056/NEJMoa1414792

8. Nogueira RG, Jadhav AP, Haussen DC, et al. Thrombectomy 6 to 24 Hours after Stroke with a Mismatch between Deficit and Infarct. N Engl J Med. 2018;378(1):11-21. doi:10.1056/NEJMoa1706442

9. Albers GW, Marks MP, Kemp S, et al. Thrombectomy for Stroke at 6 to 16 Hours with Selection by Perfusion Imaging. N Engl J Med. 2018;378(8):708-718. doi:10.1056/NEJMoa1713973

10. Bendszus M, Bonekamp S, Berge E, et al. A randomized controlled trial to test efficacy and safety of thrombectomy in stroke with extended lesion and extended time window. Int J stroke Off J Int Stroke Soc. 2019;14(1):87-93. doi:10.1177/1747493018798558

11. The TESLA Trial: Thrombectomy for Emergent Salvage of Large Anterior Circulation Ischemic Stroke - Full Text View - ClinicalTrials.gov. https://clinicaltrials.gov/ct2/show/NCT03805308. Accessed May 29, 2020.

12. MOSTE LASTE | In Extremis Study - MOSTE LASTE. https://www.inextremisstudy.com/. Accessed May 29, 2020.

13. SELECT 2: A Randomized Controlled Trial to Optimize Patient's Selection for Endovascular Treatment in Acute Ischemic Stroke - Full Text View - ClinicalTrials.gov. https://clinicaltrials.gov/ct2/show/NCT03876457. Accessed May 29, 2020.

14. Sarraj A, Hassan AE, Savitz S, et al. Outcomes of Endovascular Thrombectomy vs Medical Management Alone in Patients With Large Ischemic Cores: A Secondary 
Analysis of the Optimizing Patient's Selection for Endovascular Treatment in Acute Ischemic Stroke (SELECT) Study. JAMA Neurol. July 2019.

doi:10.1001/jamaneurol.2019.2109

15. Pizzo E, Dumba M, Lobotesis K. Cost-utility analysis of mechanical thrombectomy between 6 and 24 hours in acute ischemic stroke. Int J stroke Off J Int Stroke Soc. 2020;15(1):75-84. doi:10.1177/1747493019830587

16. Ganesalingam J, Pizzo E, Morris S, Sunderland T, Ames D, Lobotesis K. Cost-Utility Analysis of Mechanical Thrombectomy Using Stent Retrievers in Acute Ischemic Stroke. Stroke. 2015;46(9):2591-2598. doi:10.1161/STROKEAHA.115.009396

17. Xie X, Lambrinos A, Chan B, et al. Mechanical thrombectomy in patients with acute ischemic stroke: a cost-utility analysis. C open. 2016;4(2):E316-25. doi:10.9778/cmajo.20150088

18. Kunz WG, Hunink MGM, Sommer WH, et al. Cost-Effectiveness of Endovascular Stroke Therapy: A Patient Subgroup Analysis From a US Healthcare Perspective. Stroke. 2016;47(11):2797-2804. doi:10.1161/STROKEAHA.116.014147

19. Arora N, Makino K, Tilden D, Lobotesis K, Mitchell P, Gillespie J. Cost-effectiveness of mechanical thrombectomy for acute ischemic stroke: an Australian payer perspective. $J$ Med Econ. 2018;21(8):799-809. doi:10.1080/13696998.2018.1474746

20. Lobotesis K, Veltkamp R, Carpenter IH, Claxton LM, Saver JL, Hodgson R. Costeffectiveness of stent-retriever thrombectomy in combination with IV t-PA compared with IV t-PA alone for acute ischemic stroke in the UK. J Med Econ. 2016;19(8):785-794. doi:10.1080/13696998.2016.1174868 
21. Kim AS, Nguyen-Huynh M, Johnston SC. A cost-utility analysis of mechanical thrombectomy as an adjunct to intravenous tissue-type plasminogen activator for acute large-vessel ischemic stroke. Stroke. 2011;42(7):2013-2018.

doi:10.1161/STROKEAHA.110.606889

22. Sarraj A, Hassan AE, Grotta J, et al. Optimizing Patient Selection for Endovascular Treatment in Acute Ischemic Stroke (SELECT): A Prospective, Multicenter Cohort Study of Imaging Selection. Ann Neurol. 2020;87(3):419-433. doi:10.1002/ana.25669

23. CPI Home : U.S. Bureau of Labor Statistics. https://www.bls.gov/cpi/. Accessed May 29, 2020.

24. van Swieten JC, Koudstaal PJ, Visser MC, Schouten HJ, van Gijn J. Interobserver agreement for the assessment of handicap in stroke patients. Stroke. 1988;19(5):604-607. doi:10.1161/01.str.19.5.604

25. Goyal M, Menon BK, van Zwam WH, et al. Endovascular thrombectomy after largevessel ischaemic stroke: a meta-analysis of individual patient data from five randomised trials. Lancet (London, England). 2016;387(10029):1723-1731. doi:10.1016/S01406736(16)00163-X

26. Pennlert J, Eriksson M, Carlberg B, Wiklund P-G. Long-term risk and predictors of recurrent stroke beyond the acute phase. Stroke. 2014;45(6):1839-1841. doi:10.1161/STROKEAHA.114.005060

27. Arias E, Heron M, Xu J. United States Life Tables, 2014. Natl vital Stat reports from Centers Dis Control Prev Natl Cent Heal Stat Natl Vital Stat Syst. 2017;66(4):1-64. 
28. Hong K-S, Saver JL. Years of disability-adjusted life gained as a result of thrombolytic therapy for acute ischemic stroke. Stroke. 2010;41(3):471-477.

doi:10.1161/STROKEAHA.109.571083

29. Chaisinanunkul N, Adeoye O, Lewis RJ, et al. Adopting a Patient-Centered Approach to Primary Outcome Analysis of Acute Stroke Trials Using a Utility-Weighted Modified Rankin Scale. Stroke. 2015;46(8):2238-2243. doi:10.1161/STROKEAHA.114.008547

30. HCUPnet: A tool for identifying, tracking, and analyzing national hospital statistics. https://hcupnet.ahrq.gov/\#setup. Accessed May 29, 2020.

31. Shireman TI, Wang K, Saver JL, et al. Cost-Effectiveness of Solitaire Stent Retriever Thrombectomy for Acute Ischemic Stroke: Results From the SWIFT-PRIME Trial (Solitaire With the Intention for Thrombectomy as Primary Endovascular Treatment for Acute Ischemic Stroke). Stroke. 2017;48(2):379-387.

doi:10.1161/STROKEAHA.116.014735

32. Dawson J, Lees JS, Chang T-P, et al. Association between disability measures and healthcare costs after initial treatment for acute stroke. Stroke. 2007;38(6):1893-1898. doi:10.1161/STROKEAHA.106.472381

33. Chambers MG, Koch P, Hutton J. Development of a decision-analytic model of stroke care in the United States and Europe. Value Heal J Int Soc Pharmacoeconomics Outcomes Res. 2002;5(2):82-97. doi:10.1046/j.1524-4733.2002.52011.x

34. Current Population Survey (CPS) - CPS Table Creator - U.S. Census Bureau. https://www.census.gov/cps/data/cpstablecreator.html. Accessed May 29, 2020. 
35. Bureau of Labor Statistics U, Population Survey C. HOUSEHOLD DATA ANNUAL AVERAGES 3. Employment Status of the Civilian Noninstitutional Population by Age, Sex, and Race [Numbers in Thousands].; 2019.

36. Barendregt JJ, Bonneux L, van der Maas PJ. The health care costs of smoking. $N$ Engl J Med. 1997;337(15):1052-1057. doi:10.1056/NEJM199710093371506

37. Cohen JT, Neumann PJ, Weinstein MC. Does preventive care save money? Health economics and the presidential candidates. $N$ Engl J Med. 2008;358(7):661-663. doi:10.1056/NEJMp0708558 


\begin{tabular}{|c|c|c|c|}
\hline Model Input & Base-case value & Distribution & Source \\
\hline \multicolumn{4}{|l|}{$\begin{array}{l}\text { Initial probabilities for } \\
\text { achieving mRS 0/1/2/3/4/5/6 }\end{array}$} \\
\hline SELECT Large Core & & Dirichlet & Sarraj et al. \\
\hline EVT & $0.0806 / 0.0645 / 0.1613 / 0.0968 / 0.2419 / 0.0645 / 0.2903$ & & \\
\hline MM Only & $0.0233 / 0.0233 / 0.0930 / 0.1628 / 0.1628 / 0.1163 / 0.4186$ & & \\
\hline HERMES & & Dirichlet & Goyal et al. \\
\hline EVT & $0.1000 / 0.1690 / 0.1910 / 0.1690 / 0.1560 / 0.0620 / 0.1530$ & & \\
\hline MM Only & $0.0500 / 0.0790 / 0.1360 / 0.1640 / 0.2470 / 0.1350 / 0.1890$ & & \\
\hline DAWN & & Dirichlet & Nogueira et al. \\
\hline EVT & $0.0900 / 0.2200 / 0.1700 / 0.1300 / 0.1300 / 0.0900 / 0.1600$ & & \\
\hline MM Only & $0.0400 / 0.0500 / 0.0400 / 0.1800 / 0.1600 / 0.1800 / 0.1800$ & & \\
\hline DEFUSE 3 & & Dirichlet & Albers et al. \\
\hline EVT & $0.1000 / 0.1600 / 0.1800 / 0.1500 / 0.1800 / 0.0800 / 0.1400$ & & \\
\hline MM Only & $0.0800 / 0.0400 / 0.0400 / 0.1600 / 0.2700 / 0.1600 / 0.2600$ & & \\
\hline \multicolumn{4}{|l|}{ Transition probabilities } \\
\hline Recurrent stroke rate & 0.059 (for 1 st y) & Beta & Pennlert et al. \\
\hline Annual death rate & 0.013 (for $65 \mathrm{y}$ ) & Beta & Arias et al. \\
\hline $\begin{array}{l}\text { Annual death hazard rates } \\
\text { for survivors mRS } \\
0 / 1 / 2 / 3 / 4 / 5 \\
\end{array}$ & $1.53 / 1.52 / 2.17 / 3.18 / 4.55 / 6.55$ & Log normal & Hong et al \\
\hline After recurrent stroke & control arm from HERMES meta-analysis & Dirichlet & Goyal et al. \\
\hline \multicolumn{4}{|l|}{ Health care costs } \\
\hline $\begin{array}{l}\text { Costs within first } 90 \text { days } \\
\text { after stroke for mRS } \\
0 / 1 / 2 / 3 / 4 / 5 / 6 \text { (excluding IVT } \\
\text { and EVT) }\end{array}$ & $\$ 7.996 / \$ 11.038 / \$ 17.336 / \$ 21.440 / \$ 28.729 / \$ 34,319 / \$ 8,067$ & Gamma & Dawson et al. \\
\hline $\begin{array}{l}\text { Additional cost of IVT } \\
\text { treatment }\end{array}$ & ( & Gamma & NIS 2014 \\
\hline $\begin{array}{l}\text { Additional cost of EVT } \\
\text { treatment }\end{array}$ & $\$ 14,454$ & Gamma & Shireman et al. \\
\hline $\begin{array}{l}\text { Long-term annual costs } \\
\text { after stroke for mRS } \\
0 / 1 / 2 / 3 / 4 / 5\end{array}$ & $\$ 11,245 / \$ 11,579 / \$ 13,395 / \$ 23,009 / \$ 46,553 / \$ 68,441$ & Gamma & Shireman et al. \\
\hline $\begin{array}{l}\text { Recurrent stroke } \\
\text { hospitalization }\end{array}$ & 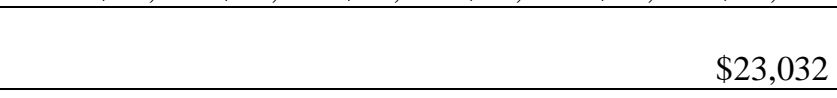 & Gamma & Chambers et al. \\
\hline \multicolumn{4}{|l|}{ Utilities } \\
\hline $\mathrm{mRS} 0 / 1 / 2 / 3 / 4 / 5 / 6$ & 1.00/0.91/0.76/0.65/0.33/0.00/0.00 & Beta & $\begin{array}{l}\text { Chaisinanunkul } \\
\text { et al. }\end{array}$ \\
\hline \multicolumn{4}{|l|}{ Societal costs } \\
\hline \multicolumn{4}{|l|}{$\begin{array}{l}\text { Paid workforce } \\
\text { productivity }\end{array}$} \\
\hline $\begin{array}{l}\text { Average annual earnings } \\
\text { of employed population }\end{array}$ & $\$ 33000$ (for $65 \mathrm{y}$ ) & Gamma & $\begin{array}{l}\text { US Census } \\
\text { Bureau } 2017\end{array}$ \\
\hline
\end{tabular}




\begin{tabular}{|l|r|l|l|}
\hline $\begin{array}{c}\text { Population employment } \\
\text { rate }\end{array}$ & 0.312 (for 65 y) & Beta & $\begin{array}{l}\text { US Bureau of } \\
\text { Labor Statistics } \\
2017\end{array}$ \\
\hline $\begin{array}{c}\text { Relative earnings of stroke } \\
\text { survivors }\end{array}$ & 0.825 & Beta & Vyas et al. \\
\hline $\begin{array}{c}\text { Return-to-work after stroke } \\
\text { mRS } 0 / 1 / 2 / 3 / 4 / 5\end{array}$ & $0.63 / 0.72 / 0.49 / 0.19 / 0.14 / 0.00$ & Beta & Tanaka et al. \\
\hline $\begin{array}{l}\text { Unpaid domestic productivity } \\
\begin{array}{c}\text { Informal annual caregiving } \\
\text { costs }\end{array}\end{array}$ & & & \\
\hline
\end{tabular}

Table 1 represents the model parameters and range of values used for sensitivity analysis. short term transition probabilities were used from SELECT ${ }^{14}$, HERMES, DAWN ${ }^{8}$ and DEFUSE $3^{9}$ population. Long term transition probabilities were based on multiple large prospective cohort studies ${ }^{25,27-29}$. Acute costs in the first 3 months include baseline medical management including adminstration of IV tPA. Utilities were derived from a validation cohort for EuroQol ${ }^{23}$. 


\begin{tabular}{|c|c|l|l|}
\hline & \multicolumn{1}{|c|}{$\begin{array}{c}\text { Endovascular } \\
\text { Thrombectomy }\end{array}$} & \multicolumn{1}{|c|}{ Medical Management } & \multicolumn{1}{c|}{ Difference } \\
\hline $\begin{array}{c}\text { Cumulative Lifetime } \\
\text { Costs }\end{array}$ & $\$ 207,866$ & $\$ 240,959$ & $\$ 33,094$ \\
\hline $\begin{array}{c}\text { Cumulative Lifetime } \\
\text { QALYs gained }\end{array}$ & 3.96 & 2.62 & 1.34 \\
\hline $\begin{array}{c}\text { Incremental Cost per } \\
\text { QALY gained }\end{array}$ & & & $\$ 24,665$ \\
\hline
\end{tabular}

Table 2 demonstrates the Costs, QALYs, ICER and NMB of EVT versus Medical treatment (MM) Based on results from patients with large core in SELECT trial. All costs are calculated in 2017 US dollars. 


\begin{tabular}{|c|c|c|c|c|}
\hline & SELECT Large Core & HERMES & DAWN & DEFUSE 3 \\
\hline Incremental Costs & $\$ 33,094$ & $-\$ 29,964$ & $-\$ 69,887$ & $-\$ 12,723$ \\
\hline Incremental QALYs & 1.34 & 1.62 & 2.36 & 2.21 \\
\hline ICER & $\$ 24,665$ & Dominant & Dominant & Dominant \\
\hline Societal Costs & $\$ 28,962$ & $-\$ 38,072$ & $-\$ 86,358$ & $-\$ 22,837$ \\
\hline
\end{tabular}

Table 3 describes the incremental costs, incremental QALY and ICERs of EVT versus MM in USD in SELECT patients with large ischemic core over horizon of lifetime, as compared to the previously published RCTs of patients with small core infarcts in both early and late time window.

The high cost of EVT was in part owing to the high mortality in MM patients in SELECT Large Core cohort, as compared to HERMES, DAWN and DEFUSE 3 cohorts. Similarly, the social cost of EVT in SELECT large core cohort was due to the reduced mortality with EVT primarily with similar rates of severe and profound disability (mRS) 4-5 between EVT and MM as compared to a difference within the same mRS grades in prior EVT RCTs. 


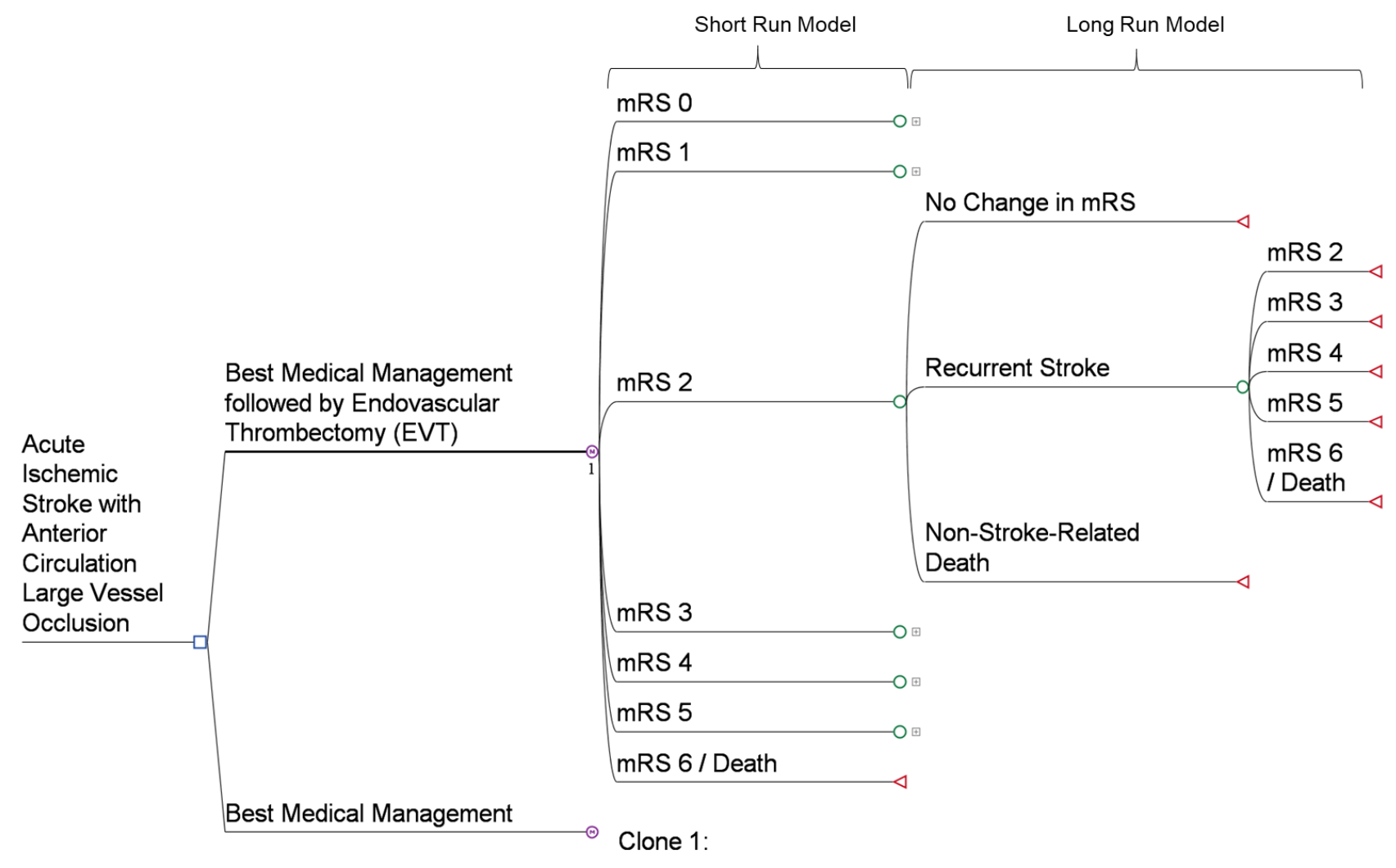

Figure 1. Decision model. A Short run analytical model is used to estimate costs within first 3 months of stroke. A long run Markov model is used to estimate transitions between states and costs beyond first 3 months of stroke. 


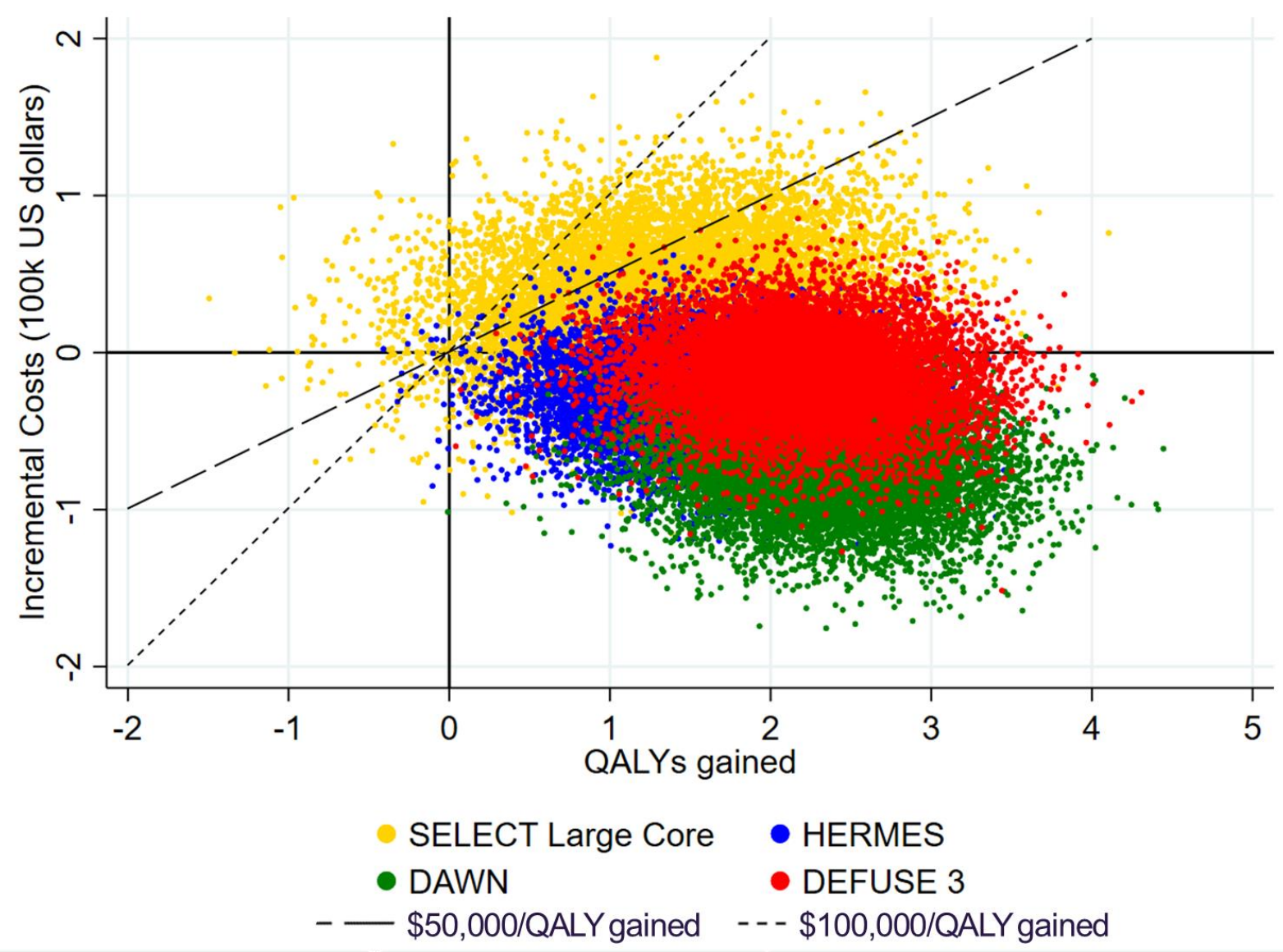

Figure 2. Results of the Probabilistic Sensitivity Analysis (PSA). The figure demonstrates 10000 simulated iterations of the incremental cost per QALY gained of EVT in patients with large core in the SELECT study (yellow), DAWN ${ }^{8}$ (green), DEFUSE3 $^{9}$ (red) and HERMES $^{25}$ (blue). The results show that the higher proportion of results are in the quadrant where an increment in costs is associated with a $Q A L Y$ gain and most of the result are under the \$50000/QALY threshold line, therefore there is a high probability that the EVT is costeffective. The long dashed line represents an ICER of \$50,000/QALY gained and short dashed line represents an ICER of $\$ 100,000 /$ QALY gained. The high cost of EVT was in part owing to the high mortality in MM patients in SELECT Large Core cohort, as compared to HERMES, DAWN and DEFUSE 3 cohorts. 


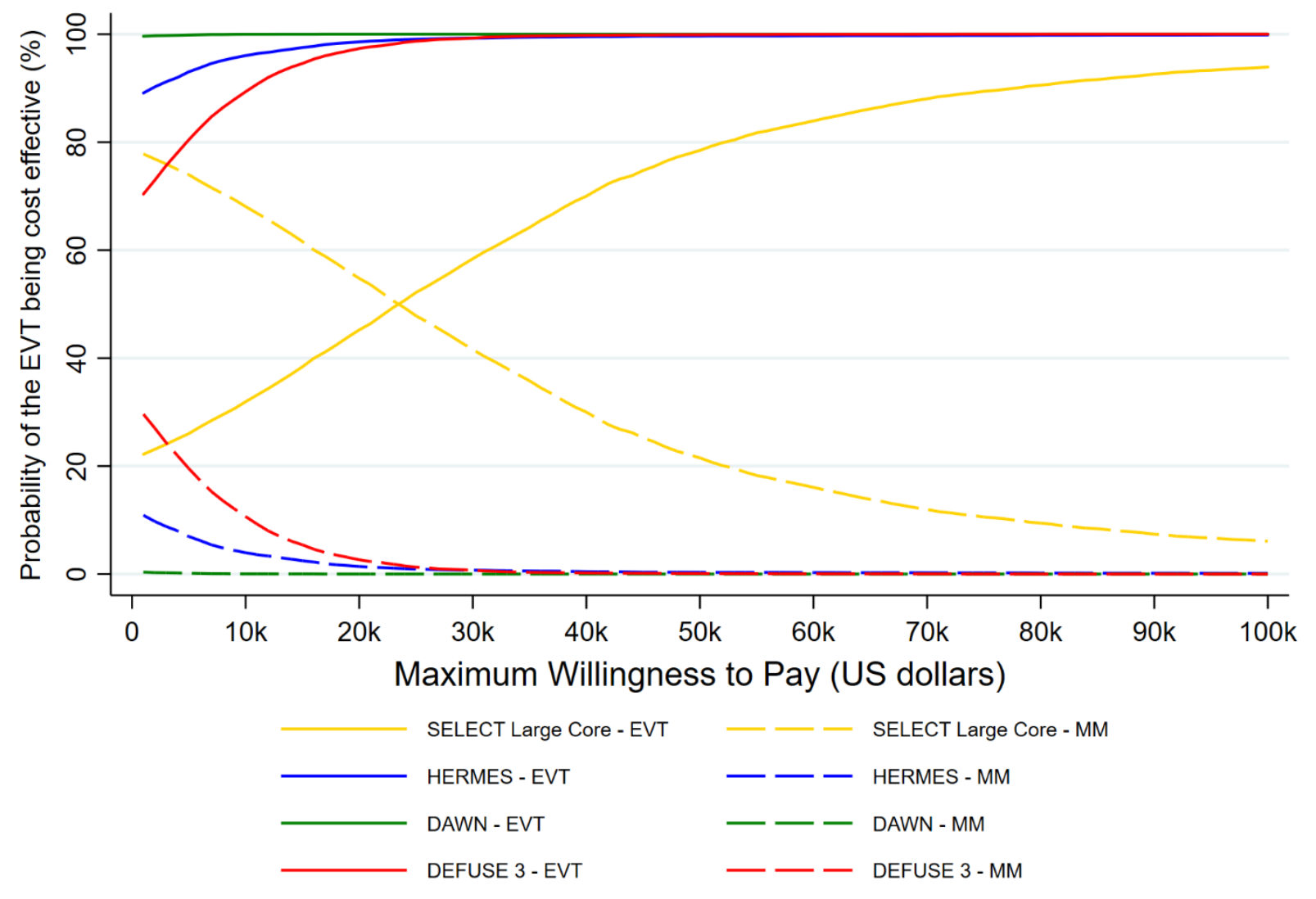

Figure 3. Cost-Effectiveness Acceptability Curves (CEAC) of EVT versus MM in the data from large core patients in SELECT trial ${ }^{14}$ (yellow), HERMES ${ }^{25}$ trial (blue), DAWN ${ }^{8}$ trial (green)and DEFUSE $3^{9}$ trial (red). The CEAC show the probability that each option is costeffective at different values of the willingness to pay for a QALY. Using SELECT data $\underline{\boldsymbol{E} V \boldsymbol{T} \boldsymbol{h a s}}$

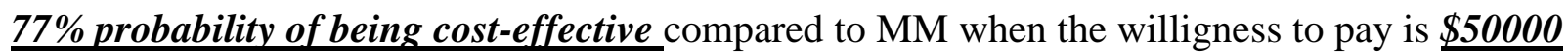
per QALY, and $92 \%$ probability of being cost-effective when the WTP is \$100000 per QALY.

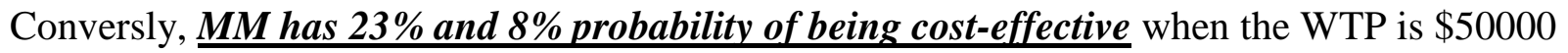
and $\$ 100000$ per QALY respectively. EVT has a probability of being cost-effective of almost $100 \%$ in all other trials with a WTP of $\$ 50,000$ and $\$ 100,000$ per QALY. The high cost of EVT 
was in part owing to the high mortality in MM patients in SELECT Large Core cohort, as compared to HERMES, DAWN and DEFUSE 3 cohorts. 